\title{
Distributed-Feedback Grating Used as a Lateral- Mode Selector in Phase-Locked Antiguided Arrays
}

\author{
M. P. Nesnidal, L. J. Mawst, Senior Member, IEEE, and D. Botez, Fellow, IEEE
}

\begin{abstract}
We show here, for the first time, that a distributedfeedback (DFB) grating can act as a lateral-mode discriminator if located below the active region of a phase-locked antiguided array. Spatial-mode selection from such a lower-DFB (LDFB) grating in a resonant antiguided structure (ROW-LDFB) relies on the fact that the optical field distribution below the active region is strongly array-mode dependent. In particular, it is shown that at and near resonance a ROW-LDFB structure strongly favors resonant-mode oscillation, while suppressing oscillation of highorder modes. ROW-LDFB devices thus accomplish both spatialand frequency-mode selection in a single structure. Furthermore, for effective intermodal discrimination, there is no need for interelement loss or Talbot-type filters, thus eliminating all potential sources of self-pulsations.
\end{abstract}

Index Terms - Semiconductor laser arrays, spatial filters, phase-locked oscillators, distributed feedback lasers.

T THE ABILITY to produce high-power, single-frequency, single-spatial-mode light sources is of fundamental importance to many optoelectronic applications. Fanout-type master-oscillator power-amplifier (MOPA) devices of the "broad-area" type have displayed watts of diffraction-limited, single-frequency power [1]-[3]. However, broad-area MOPA devices possess inherent instabilities due to refractive-index variations induced by thermal gradients and injected carriers [3]-[6], and are highly sensitive to feedback [7], thus raising issues of long-term stability and reliability. Therefore, there is a need for large-aperture devices with strong built-in index guiding.

Large-aperture phase-locked arrays of resonant antiguides (so-called ROW arrays) have demonstrated the unique ability to operate in-phase with stable, diffraction-limited beams to record-high pulsed and CW output powers [8], [9]. We have demonstrated [10] the first simultaneous single-frequency and single-spatial-mode operation from resonant antiguided arrays employing distributed feedback (i.e., ROW-DFB arrays). A second-order DFB grating located above the active layer provided frequency discrimination, while InGaAs quantum wells within high-index GaAs interelement regions provided interelement loss for spatial-mode selectivity. However, it has been shown [11] that interelement field could cause selfpulsations due to saturable absorption at high drive levels. One can avoid the need for interelement loss by using Talbot-type

Manuscript received July 26, 1996; revised September 19, 1996. This work was supported in part by the National Science Foundation under Grant ECS9522035

The authors are with the Electrical Engineering Department, University of Wisconsin-Madison, 1415. Engineering Drive, Madison, WI 53706 USA

Publisher Item Identifier S 1041-1135(97)00582-X

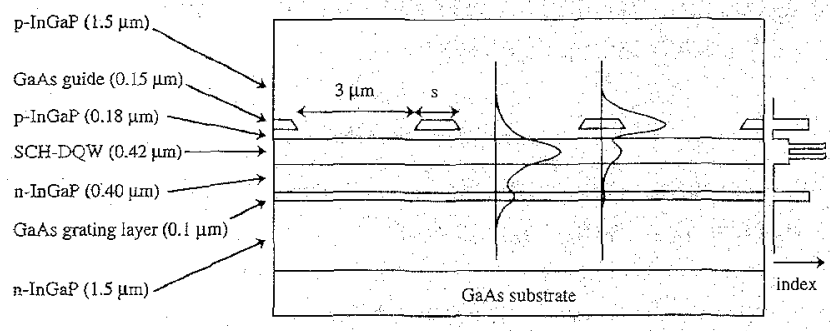

Fig. 1. Cross-sectional schematic of a ROW-LDFB array in which a lower DFB grating is used as a spatial-mode selector. Transverse element and interelement field profiles, and their overlap with the grating layer, illustrate that the lower DFB preferentially amplifies array-modes with significant optical field in the element regions.

spatial filters [12], but this method could also cause instabilities [13] and/or multimoding [14] at high powers. Therefore, in order to increase the maximum stable, coherent power from index-antiguided devices, there is a need for a novel spatialmode selector that is insensitive to drive level and/or thermally induced index gradients.

Several attempts [15]-[17] to use DFB gratings for arraymode discrimination in phase-locked arrays have failed, since modes adjacent to the in-phase mode have phasefronts virtually identical to that of the in-phase mode. We show here, however, that a DFB grating can actually serve as a spatialmode selector in antiguided arrays if located below the active layer. Spatial-mode discrimination from such ROW arrays with lower DFB gratings (ROW-LDFB) relies on the fact that the array mode which interacts strongest with the grating, and thus has preferential feedback, is the resonant mode.

ROW arrays have been previously analyzed [12] A A schematic representation of the structure studied here is shown in Fig. 1. Optical confinement and the necessary gain are provided by a SCH-DQW structure consisting of $2000-\AA$ thick InGaAsP confinement layers surrounding two $70-\AA$-thick InGaAs quantum wells that are separated by a $100-\AA$-thick InGaAsP barrier. Upper and lower InGaP cladding layers are $1.5 \mu \mathrm{m}$ thick. A second-order sinusoidal DFB grating, with a tooth height of $1000 \AA$, is defined atop the lower cladding layer and separated from the lower confinement layer by a $4000-\AA$-thick InGaP spacer layer. The (high-effectiveindex) interelement regions are defined by $1500-\AA$-thick GaAs passive guide regions separated from the upper confinement layer by a $1800-\AA \AA$-thick InGaP layer, yielding an effectiveindex step between regions of 0.026 at resonance. From transverse element and interelement field profiles (Fig. 1) one can easily see that the element field has a greater overlap with the grating layer compared to the interelement field. As a 
result, array modes with most field in the elements experience strongest optical feedback. Moreover, since the in-phase array mode is nearly resonant within $200-300 \AA$ of the longitudinal resonance [9], the ability to simultaneously satisfy the lateral and longitudinal resonance conditions is not very sensitive to variations in grating period about the longitudinal resonance.

The grating geometry and its location were chosen such that the normalized coupling coefficients in the element and interelement regions, $\kappa_{e} L$ and $\kappa_{i} L$, are $\approx 3$ and 0.5 , respectively. 10 -element, $350-\mu \mathrm{m}$-long devices are designed to operate near the in-phase mode resonance. Element widths of $3 \mu \mathrm{m}$ are considered, while the interelement width, $s$, is varied to determine optimal discrimination between the inphase mode and competing modes. The normalized coupling coefficients in these two regions were determined using the method presented by Streifer et al. [18] for sinusoidal gratings. A normalized overall coupling coefficient, $\kappa L$, can then be constructed from

$$
\kappa L=\kappa_{e} L\left(\Gamma_{L}\right)+\kappa_{i} L\left(1-\Gamma_{L}\right)
$$

where $L$ is the device length; $\kappa_{e}$ and $\kappa_{i}$ are element and interelement coupling coefficients, respectively; and $\Gamma_{L}$ is the fraction of the optical field residing in the element regions. $\Gamma_{L}$ was determined by calculating the array-mode losses using the effective-index approximation [12] with and without interelement loss in each case. The amount of interelement field, and thus $\Gamma_{L}$, can then be determined by subtraction. Although $\kappa L$ and $\Gamma_{L}$ are obtained by using a one dimensional (1-D) approximation, the trends in array-mode coupling to the grating for the more realistic two-dimensional (2-D) case [9] are expected to be similar. The determination of the coupling coefficient in (1) is an iterative process whereby one varies the transverse element or interelement structure and solves for the fundamental transverse modes, calculates element and interelement coupling coefficients, and determines an overall coupling coefficient until the desired value of $\kappa L$ is achieved. $\kappa L$ is selected such that feedback due to the DFB grating will dominate Fabry-Perot contributions. We seek values within the range $1<\kappa L<5$, neglect facet reflectivities, and assume that the cavity length is an integral multiple of the grating period. Once the transverse structure is determined, the edge radiation loss, $\alpha_{\text {rad }}$, and the 2-D field overlap with the active layer(s), $\Gamma_{2-D}[19]$, are numerically calculated as a function of $s$ for the in-phase and competing modes.

Calculation of an effective mirror loss, $2 \gamma$, for the grating can be accomplished by considering the reflection gain [20]

$$
\frac{E_{r}(0)}{E_{i}(0)}=\frac{\kappa \sinh S L}{(\gamma-i \Delta \beta) \sinh S L-S \cosh S L}
$$

where $\kappa$ is the overall coupling coefficient, $\Delta \beta$ is the deviation of the propagation constant from the Bragg condition, and $S^{2}=\kappa^{2}+(\gamma-i \Delta \beta)^{2}$. For a given $\kappa$ value, the longitudinal modes are characterized by pairs of $\gamma$ and $\Delta \beta$ that yield infinite gain singularities in (2). Note that since $\kappa$ is spatial-modedependent from (1), so too is the effective mirror loss. The grating therefore acts as spatial-mode-dependent end-mirrors.

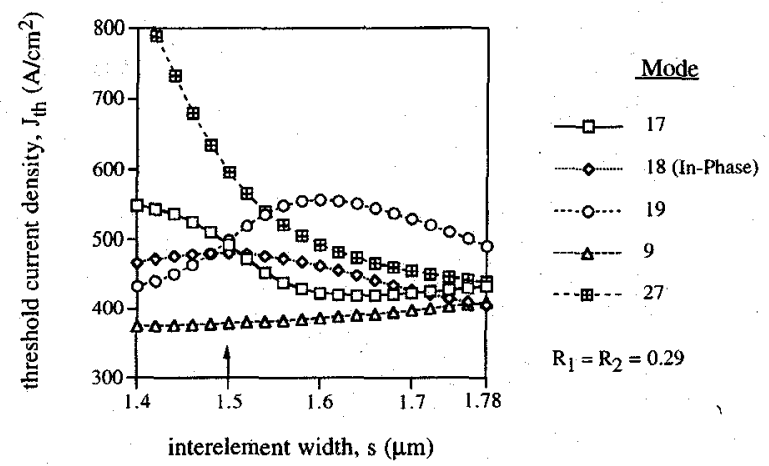

Fig. 2. Threshold current density versus interelement width for a ROW-FP array. Optical feedback is provided by the cleaved facets. The in-phase mode 18 resonance is indicated by the arrow.

The bulk threshold gain, $g_{\mathrm{th}, m}$, and threshold current densities, $J_{\mathrm{th}, \dot{m}}$, for mode $m$ are

$$
\begin{aligned}
& g_{\mathrm{th}, m}=\left(2 \gamma_{m}+\alpha_{\mathrm{rad}, m}\right) / \Gamma_{2 \mathrm{D}, m} \\
& J_{\mathrm{th}, m}=J_{o}+\frac{g_{\mathrm{th}, m}}{B}
\end{aligned}
$$

where $J_{o} \approx 150 \mathrm{~A} / \mathrm{cm}^{2}$ is the transparency current density and $B \approx 4.5 \mathrm{~cm} / \mathrm{A}$ is the gain coefficient. In (3), the internal cavity loss is assumed to be negligible.

For the structure described in Fig. 1, the normalized overall coupling coefficients were calculated for in-phase mode $m=$ 18 (see [12]), adjacent modes 17 and 19, and out-of-phase modes 9 and 27 to be: $4.11,1.91,4.08,2.34$, and 3.41 , respectively. $\kappa L$ for the in-phase mode 18 was not found to be a strong function of $\mathrm{s}$. The range of $\mathrm{s}$ considered here is from $1.40-1.78 \mu \mathrm{m}$, while the in-phase mode resonance occurs for $s=1.5 \mu \mathrm{m}$.

To determine the effect of the lower grating, thresholdcurrent densities of the competing modes, $J_{\mathrm{th}, m}$, were calculated as a function of the interelement width, $s$, (Fig. 2) for arrays employing only Fabry-Perot cavities (ROW-FP) for which mirror reflectivities are assumed to be $R_{1}=R_{2}=0.29$. It is clear that out-of-phase mode 9 is favored to lase for most of the range in $s$, despite the fact that the in-phase mode has the highest $\Gamma_{2-D}$ value of all modes at and near its resonance [19]. That is, the difference in edge radiation losses between modes overcomes their difference in $\Gamma_{2-D}$ to favor lasing of the outof-phase mode 9 . This fact becomes clear by plotting $\alpha_{\mathrm{rad}, m}$ versus $s$ (Fig. 3). Compared to the in-phase mode, $\alpha_{\mathrm{rad}}$ is negligible for mode 9 over the entire range in $s$, while $\alpha_{\text {rad }}$ for adjacent mode 17 is relatively low at and above resonance, as expected from [12]. The conclusion to be drawn from Figs. 2 and 3 is that the modal edge radiation loss, $\alpha_{\mathrm{rad}, m}$, is the dominant spatial-mode discriminator for 10-element ROWFP structures, resulting in lasing of the out-of-phase mode 9 over a wide range in $s$. (In conventional 10-element ROWFP devices, mode 9 is suppressed by using interelement loss and/or Talbot-type spatial filters [12].)

For ROW-LDFB arrays, $\alpha_{\text {rad, } m}$ (Fig. 3) and $\Gamma_{2 \text {-D }}$ closely resemble the behavior of ROW-FP devices. The array-modedependent interaction with the lower grating, which favors array modes with significant field in the element regions, counteracts the radiation loss role as a spatial-mode discriminator 


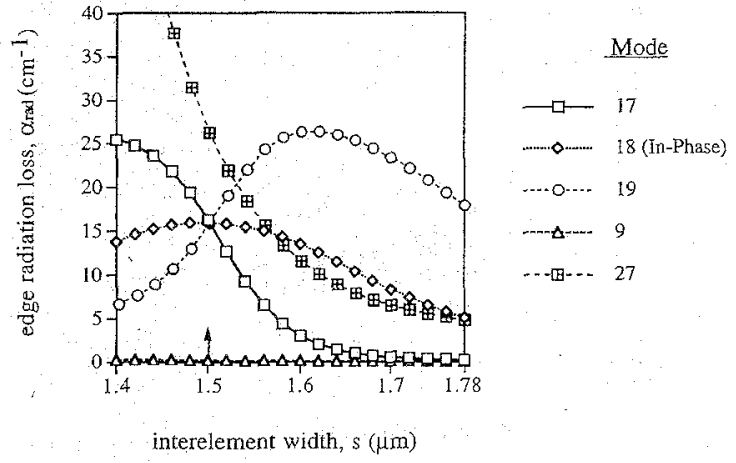

Fig. 3. Modal radiation loss versus interelement width for a ROW-FP array. The in-phase mode 18 resonance is indicated by the arrow.

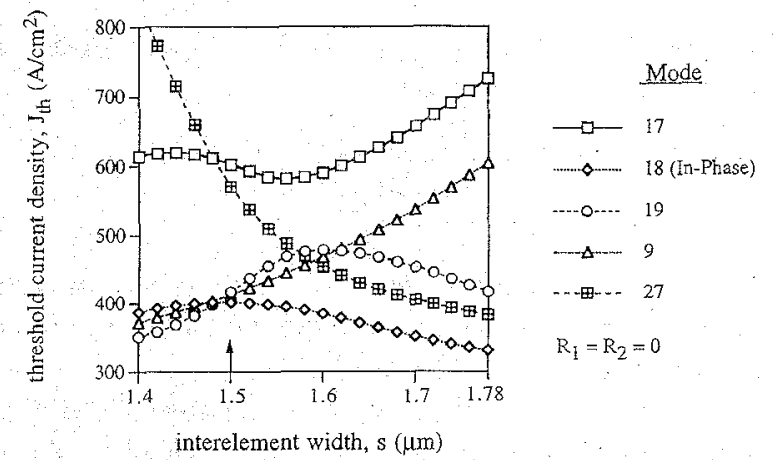

Fig. 4. Threshold current density versus interelement width for a ROW-LDFB array as shown in Fig. 1. Array-mode-sensitive feedback is provided by the lower DFB grating. The in-phase mode 18 resonance is indicated by the arrow.

(3). In-phase mode operation from ROW-LDFB structures, therefore, is possible provided the lateral structure in Fig. 1 is designed such that the low effective mitror loss, $2 \gamma$, for in-phase mode 18 sufficiently offsets its high $\alpha_{\text {rad }}$ value compared to all other competing modes.

Threshold-current densities calculated for this structure are shown in Fig. 4. Compared to Fig. 2, the effectiveness of the lower grating as an in-phase array-mode selector is clearly seen. (It should be noted that the effects of surface radiation from second-order gratings impact similarly modes 18 , 19 , and 27 due to the comparable amount of element field [12]. As a result, discrimination resembling that displayed in Fig. 4 is still expected. The minimization of surface emission through grating geometry optimization is left to future work.) The threshold-current densities for out-of-phase mode 9 and adjacent-mode 17 dramatically increase with $s$. This behavior can be understood as a consequence of those modes' decreasing field in the array elements with increasing s [12], resulting in less interaction with the grating region (see Fig. 1). By contrast, in-phase mode 18 has significant element field and strongly couples to the grating region. As a result, in ROWLDFB arrays, in-phase mode operation is favored at and above its resonance.

It has been theoretically shown, for the first time, that a DFB grating can act as a spatial-mode discriminator if located below the active region in a ROW array.

\section{ACKNOWLEDGMENT}

The authors would like to thank Dr. J. Buus of Gayton Photonics for his guidance in this work.

\section{REFERENCES}

[1] J. N. Walpole, E. S. Kintzer, S. R. Chinn, C. A. Wang, and L. J. Missaggia, "High-power strained-layer InGaAs/AlGaAs tapered traveling wave amplifier," Appl. Phys. Lett., vol. 61, pp. 740-742, Aug. 1992

[2] D. F. Welch, R. Parke, D. Mehuys, A Hardy, R Lang, S O'Brien, and D. Scifres, " $1,1 \mathrm{~W} \mathrm{CW}$, diffraction-limited operation of a monolithically integrated flared-amplifier master oscillator power amplifier," Electron. Lett., vol. 28, pp. 2011-2013, 1992 .

[3] D. Mehuys, L. Goldberg, and D. F. Welch, $45.25-\mathrm{W}$ CW neardiffraction-limited tapered-stripe semiconductor optical amplifier," IEEE Photon. Technol. Lett., vol. 5, pp. 1179-1182, Oct, 1993.

[4] J. H. Abeles; R. Amantea R. Rios, and D. J Channin; "Finite difference beam propagation method modeling for high power fanned-out amplifier lasers," presented at the OSA Optical Design for Photonics Conf., Palm. Springs, CA., Mar. 22-24, 1993, paper PD-2.

[5] L. Goldberg, M. Surette, and D. Mehuys, "Filament formation in a tapered GaAlAs optical amplifier," Appl. Phys. Lett., vol. 62, no: 5, pp. $2304-2306,1993$

[6] S. Ramanujan and H. G. Winful, "Spontaneous emission induced filamentation in flared amplifiers," IEEE Y. Quantum Electron, vol. 32, pp. 784 789, May 1996

[7] D. J. Bossert, J. R. Marciante, and M. W. Wtight, Feedback effects in tapered broad-area semiconductor lasers and amplifiers" IEEE Photon. Technol. Lett, vol. 7, pp. 470-472, May 1995 .

[8] L. J. Mawst, D. Botez, C. Zmudzinski, M. Jansen, C. Tu, T. J. Roth and J. Yun, "Resonant self-aligned-stripe antiguided diode laser array," Appl. Phys. Lett., vol. 60, pp. 668-670, 1992.

[9] D. Botez, "High-power monolithic phase-locked arrays of antiguided semiconductor diode lasers," Proc. Inst. Elect. Eng. Pt J., Optoelectron. vo1. 139, pp. 14-23, Feb, 1992

[10] M. P. Nesnidal, L. J. Mawst, A. Bhattacharya, D. Botez, L. DiMarco, J. C. Conrolly, and J. H. Abeles, "Single-frequency, single-spatial-mode ROW-DFB diode laser arrays," IEEE Photon Technol. Lett., Yol. 8, pp. 182-184, Feb. 1996.

[11] S. Ramanujan, H. G. Winful, M. Felsky, R. K. DeFreez D. Botez M. Janser, and P. Wisseman, "Temporal behavior of tesonant-opticalwaveguide phase-locked diode laser arrays," Appl. Phys. Lett., vol. 64, no. 7 , pp. $827-829$. Feb. 1994

[12] D. Botez, L. J. Mawst, G. L. Peterson, and T. J. Roth, "Phaselocked arrays of antiguides: modal content and discrimination," IEEEJ. Quantum Electron, vol. 26, pp. 482 495, Mar. 1990.

[13] R. F. Nabiev, M. D. Iturbe-Castillo, J. J. Sanchez-Mondragon, A. I Onishchenko, and D. Botez, Nonlinear restrictions of Talbot-like spatial filters in semiconductor laser arrays, Appl. Opt, vol. 32, no. 24, pp 4480 4484, Aug. 1993.

[14] C. Zmudzinski, D. Botez, and L.J. Mawst, "Coherent, one-watt operation of large-aperture resonant arrays of antiguided diode lasers," Appl. Phys Lett., vol. 62, pp. 2914 2916, June 1993.

[15] K.-Y. Liou, A. G. Dentai, E. C. Burrows, R. P. Gnall, C. M. Joyner, and C. A. Burrus, "High-power multiple-quantum-well distributed feedback laser arrays and Fabry-Perot laser arrays at $1.5 \mu \mathrm{m}$ wavelength," in Tech. Dig. 13th IEEE Int. Semiconductor Laser Conf., Takamatsu, Japan, Sept. 21-25, pp. 88-89, 1992, paper D-27:

[16] L. M. Miller, K. J. Beernink, J. T. Verdeyen, J. J, Coleman, J. S Hughes, G. M. Smith, J. Honig, and T. M. Cockerill, "InGaAs-GaAsAlGaAs strained-layer distributed feedback ridge waveguide quantum well heterostructure Iaser array, "'. Electron. Lett., vol 27, pp. 1943-1945, 1991.

[17] Y. Twu, N. K. Dutta, C. A. Green, and J. D. Wynn, "GaInAsP distributed feedback laser array," Electron. Lett., vol. 24, pp. 743-744, 1988.

[18] W. Streifer, D. R. Scifres, and R. D. Burnham, "Coupling coefficients for distributed feedback single and double-heterostncture diode lasers," IEEE J. Quantum Electron., vol. 11, pp 867-873, Nov. 1975.

[19] D. Botez and L. J Mawst, "T effect: Key intermodal-discrimination. mechanism in arrays of antiguided diode lasers," Appl Phys Lett, vel. 60, pp. 3096-3098, June 1992.

[20] P. Yeh, Optical Waves in Layered Media. New York: Wiley, 1988 